\title{
Erratum to: Optimal Fuzzy Controller Design Using an Evolutionary Strategy-Based Particle Swarm Optimization for Redundant Wheeled Robots
}

\author{
Hsu-Chih Huang ${ }^{1}$ Sendren Sheng-Dong $\mathrm{Xu}^{2} \cdot$ Chih-Hao Chiang ${ }^{1}$
}

Published online: 14 May 2016

(C) Taiwan Fuzzy Systems Association and Springer-Verlag Berlin Heidelberg 2016

\section{Erratum to: Int. J. Fuzzy Syst. (2015) 17(3):390-398}

DOI 10.1007/s40815-015-0055-y

In the original publication of this article Fig. 4 is incorrectly published. This error in no way affects the conclusions of the study. The correct figure is provided in this erratum.

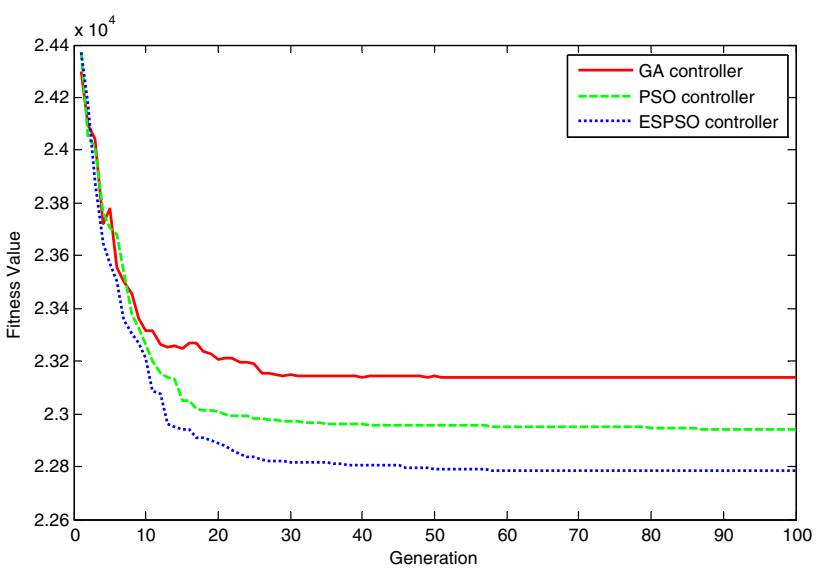

Fig. 4 The evolutions of the FC-ESPSO and conventional controllers to achieve elliptic trajectory tracking

The online version of the original article can be found under doi:10.1007/s40815-015-0055-y.

Hsu-Chih Huang

hchuang@niu.edu.tw

Sendren Sheng-Dong Xu

sdxu@mail.ntust.edu.tw

1 Department of Electrical Engineering, National Ilan University, Yilan, Taiwan

2 Graduate Institute of Automation and Control, National Taiwan University of Science and Technology, Taipei, Taiwan 